\title{
A MAXIMUM PRINCIPLE FOR COMPRESSIBLE FLOW ON A SURFACE
}

\author{
LESLEY M. SIBNER AND ROBERT J. SIBNER
}

\begin{abstract}
We show that the speed of a steady, irrotational, subsonic flow on a surface cannot attain its maximum at a point of positive Gauss curvature.
\end{abstract}

In his work on curvature and homology, Bochner [3] obtained a formula for the Laplacian of the norm of a harmonic form on an orientable Riemannian manifold in terms of the curvature of the manifold. In this paper we obtain a corresponding formula for $\rho$-harmonic forms which describe compressible flows and will use this result to show that a steady, irrotational subsonic fluid flow on a surface cannot attain its maximum speed at a point of positive Gaussian curvature.

1. Compressible flows on a surface. In the representation of a steady flow on an orientable surface $M$ by a differential 1 -form $\omega=\omega_{1} d x+\omega_{2} d y$, the requirement that the flow be irrotational (no circulation about curves homologous to zero) is expressed by the first order differential equation $d \omega=0$ when $d$ is the exterior derivative. One says that the form is closed and this is equivalent to the existence locally of a single valued potential function. The requirement of conservation of mass leads to a second first order equation $\delta(\rho \omega)=0$ where $\delta$ is the adjoint of $d$ and $\rho$ is the density. We shall recall the explicit expressions for these partial differential operators in $\$ 3$ and only remark here that $\delta$, unlike $d$, depends on the Riemannian metric $g_{i j}$ which we assume given on $M$.

We have called [5] a form $\omega$ satisfying the system

$$
d \omega=0, \quad \delta \rho \omega=0,
$$

a $\rho$-harmonic form. If $\rho$ is constant then the flow is incompressible and the system (1) simply expresses the well-known fact that an incompressible flow is described by a harmonic form.

2. Subsonic flows. Letting $\langle\omega, v\rangle=g^{i j} \omega_{i} v_{j}$ denote the (pointwise) inner product induced by the Riemannian metric, we refer, for convenience, to the square of the norm $Q=\langle\omega, \omega\rangle$ as the speed of the flow. From physical considerations we make the rather general assumptions (cf. [1]) that the density $\rho$ of the fluid is a function of $Q$ alone which is bounded above and

Received by the editors November 10, 1977.

AMS (MOS) subject classifications (1970). Primary 35J60, 53B20. 
below by positive constants and is such that the "mass velocity" is an increasing function of the speed for speeds below some critical speed. Specifically, in terms of $Q$, this is expressed by

$$
\begin{aligned}
& \frac{d}{d Q}\left(\rho^{2} Q\right)>0 \text { for } Q<Q_{s}, \\
& \frac{d}{d Q}\left(\rho^{2} Q\right) \rightarrow 0 \quad \text { as } Q \nearrow Q_{s} .
\end{aligned}
$$

The quantity $Q_{s}$ is called the sonic speed. At each point the flow (and the form representing it) is said to be subsonic, sonic or supersonic according as $Q(\omega)<Q_{s}, Q(\omega)=Q_{s}$ or $Q(\omega)>Q_{s}$.

Global existence, uniqueness and regularity questions for subsonic flows on compact manifolds and on compact manifolds with boundary have been discussed in other work [5], [7].

Generally speaking, as the "data" increases, the maximum speed attained by a subsonic flow increases towards the sonic speed $Q_{s}$. The location of the point(s) where the maximum speed is attained is thus clearly of some interest. In particular, given a (smooth) subsonic flow on a surface, one can ask whether there are differential geometric restrictions on the locations of points where the flow can attain its maximum speed. In what follows, we will verify a conjecture, made in [6] that the maximum speed of a subsonic compressible flow cannot be attained at an interior point of positive Gaussian curvature.

3. The fundamental formula. In terms of covariant derivatives $\nabla_{i}$ we can write explicit local expressions for $d$ and $\delta$. Since we deal only with manifolds of dimension 2 , we need only describe their action on functions $f, 1$-forms $\zeta=\zeta_{i} d x^{i}$ and 2-forms $\Phi=\varphi_{i j} d x^{i} \wedge d x^{j}(i, j=1,2)$.

$$
\begin{array}{llrl}
d f=\nabla_{i} f d x^{i}=\frac{\partial f}{\partial x^{i}} d x^{i}, & d \zeta=-\frac{1}{2}\left(\nabla_{j} \zeta_{i}-\nabla_{i} \zeta_{j}\right) d x^{i} \wedge d x^{j}, & d \Phi=0, \\
\delta f=0, & \delta \zeta=-g^{i j} \nabla_{i} \zeta_{j}, & \delta \Phi=-g^{i j} \nabla_{i} \varphi_{j k} d x^{k} .
\end{array}
$$

Recall also that the Laplace-Beltrami operator (on functions or forms) is defined by

$$
\Delta=d \delta+\delta d
$$

Applied to functions, this can be written

$$
\Delta f=-g^{j k} \nabla_{k} \nabla_{j} f=-\frac{1}{\sqrt{g}} \frac{\partial}{\partial x^{i}}\left(\sqrt{g} g^{i j} \frac{\partial f}{\partial x^{j}}\right) \text { where } g=\operatorname{det} g_{i j} \text {. }
$$

For a form which is not necessarily harmonic, a modification of Bochner's formula can be obtained as follows.

Since $Q=g^{a b} \zeta_{a} \zeta_{b}$, a direct computation yields

$$
\begin{aligned}
\Delta Q & =-g^{i j} \nabla_{i} \nabla_{j} Q=-g^{i j} \nabla_{i}\left(2 g^{a b} \zeta_{a} \nabla_{j} \zeta_{b}\right) \\
& =-2 g^{i j} g^{a b}\left(\nabla_{i} \zeta_{a}\right)\left(\nabla_{j} \zeta_{b}\right)-2 g^{i j} g^{a b} \zeta_{a} \nabla_{i} \nabla_{j} \zeta_{b} .
\end{aligned}
$$

Writing $|\nabla \zeta|$ for the norm (induced by the Riemannian metric) of the tensor 
$\nabla_{k} \zeta_{l}$ we obtain

$$
-\frac{1}{2} \Delta Q=|\nabla \zeta|^{2}+g^{i j} g^{a b} \zeta_{a} \nabla_{i} \nabla_{j} \zeta_{b}
$$

On the other hand,

$$
\Delta \zeta=d \delta \zeta+\delta d \zeta=-g^{i j} \nabla_{i} \nabla_{j} \zeta_{b} d x^{b}+g^{k j}\left(\nabla_{k} \nabla_{b} \zeta_{j}-\nabla_{b} \nabla_{k} \zeta_{j}\right) d x^{b}
$$

which, by the Ricci identity, can be written in terms of the Gaussian curvature $K$

$$
\Delta \zeta=-g^{i j} \nabla_{i} \nabla_{j} \zeta_{b} d x^{b}+K \zeta_{b} d x^{b}
$$

so that

$$
\langle\Delta \zeta, \zeta\rangle=-g^{a b} \zeta_{a} g^{i j} \nabla_{i} \nabla_{j} \zeta_{b}+K\langle\zeta, \zeta\rangle
$$

Combining (4) and (5) we obtain finally

$$
-\frac{1}{2} \Delta Q(\zeta)+\langle\Delta \zeta, \zeta\rangle=|\nabla \zeta|^{2}+K Q
$$

4. The speed of a compressible flow. If (6) is applied to a form $\zeta=\rho \omega$, where $\rho=\rho(Q(\omega))$, and it is observed that $Q(\rho \omega)=\rho^{2} Q(\omega)=\rho^{2} Q$, we obtain

$$
-\frac{1}{2} \Delta \rho^{2} Q+\langle\Delta \rho \omega, \rho \omega\rangle=|\nabla \rho \omega|^{2}+\rho^{2} K Q .
$$

We now express the left-hand side of (7) as an operator on $Q$.

Writing $-\frac{1}{2} \Delta \rho^{2} Q=-\frac{1}{2}\left(\rho^{2}+2 \rho \rho^{\prime} Q\right) \Delta Q+a_{0}(x, y) Q_{x}+b_{0}(x, y) Q_{y}=$ $L_{1} Q$ we observe that the positivity of the term in parentheses is precisely the condition (2a) that the flow be subsonic. Consequently, for such a flow $L_{1}$ is uniformly elliptic.

Assuming now that $\omega$ is $\rho$-harmonic and using (1) we can write $\Delta \rho \omega=\delta d \rho \omega$ $=\delta\left(\rho^{\prime} d Q \wedge \omega\right)$. Assuming further that we have chosen isothermal coordinates, so that $g_{i j}=\lambda \delta_{i j}$, we have

$$
\begin{aligned}
\Delta \rho \omega= & \delta\left(\left[\rho^{\prime} \omega_{2} Q_{x}-\rho^{\prime} \omega_{1} Q_{y}\right] d x d y\right) \\
= & {\left[\left(\frac{\rho^{\prime} \omega_{2}}{\lambda} Q_{x}\right)_{y}-\left(\frac{\rho^{\prime} \omega_{1}}{\lambda} Q_{y}\right)_{y}\right] d x+\left[\left(\frac{\rho^{\prime} \omega_{1}}{\lambda} Q_{y}\right)_{x}-\left(\frac{\rho^{\prime} \omega_{2}}{\lambda} Q_{x}\right)_{x}\right] d y } \\
= & {\left[\frac{\rho^{\prime} \omega_{2}}{\lambda} Q_{x y}-\frac{\rho^{\prime} \omega_{1}}{\lambda} Q_{y y}+a_{1} Q_{x}+b_{1} Q_{y}\right] d x } \\
& +\left[\frac{\rho^{\prime} \omega_{1}}{\lambda} Q_{y x}-\frac{\rho^{\prime} \omega_{2}}{\lambda} Q_{x x}+a_{2} Q_{x}+b_{2} Q_{y}\right] d y
\end{aligned}
$$

where (for a fixed form $\omega$ ) the coefficients $a_{i}$ and $b_{i}$ are functions of $x$ and $y$. We have then

$$
\begin{aligned}
\langle\Delta \rho \omega, \rho \omega\rangle= & -\frac{\rho \rho^{\prime}}{\lambda^{2}}\left[\omega_{2}^{2} Q_{x x}-2 \omega_{1} \omega_{2} Q_{x y}+\omega_{1}^{2} Q_{y y}\right] \\
& +a(x, y) Q_{x}+b(x, y) Q_{y} \\
= & L_{2} Q .
\end{aligned}
$$

In order to investigate the ellipticity of the operator $L=L_{1}+L_{2}$, we 
distinguish the two cases $\rho^{\prime}(Q) \leqslant 0$ and $\rho^{\prime}(Q)>0$. In the former case (which is the more interesting physically) it is immediately seen that $L_{2}$ is degenerate elliptic and since $L_{1}$ is uniformly elliptic it follows that $L$ is uniformly elliptic. If, on the other hand, $\rho^{\prime}>0$ we write

$$
\begin{aligned}
L_{1} Q & =-\frac{1}{2} \rho^{2} \Delta Q-\rho \rho^{\prime} Q \Delta Q \\
& =-\frac{1}{2} \rho^{2} \Delta Q+\rho \rho^{\prime}\left[\lambda^{-1}\left(\omega_{1}^{2}+\omega_{2}^{2}\right)\right]\left[\lambda^{-1}\left(Q_{x x}+Q_{y y}\right)\right] .
\end{aligned}
$$

Then the principal part of $\left(L_{1}+L_{2}\right) Q$ is

$$
-\frac{1}{2} \rho^{2} \Delta Q+\frac{\rho \rho^{\prime}}{\lambda^{2}}\left(\omega_{1}^{2} Q_{x x}+2 \omega_{1} \omega_{2} Q_{x y}+\omega_{2}^{2} Q_{y y}\right) .
$$

The first term is uniformly elliptic and the second degenerate elliptic, so that again $L$ is uniformly elliptic. In either case we have the

LEMmA 1. The speed $Q$ of a $\rho$-harmonic form $\omega$ satisfies a second order partial differential equation

$$
L Q=|\nabla \rho \omega|^{2}+\rho^{2} K Q
$$

If $\omega$ is subsonic, then $L$ is uniformly elliptic. If $K \geqslant 0$ on an open set $U$, then $Q$ is a subsolution $(L Q \geqslant 0)$ of $L$ on $U$.

The equation (8) is the $\rho$-harmonic analogue of Bochner's fundamental formula for harmonic forms.

LEMMA 2. If $Q \neq 0$ for a subsonic $\rho$-harmonic form $\omega$ on $M$ then the interior zeros of $Q$ are isolated.

Proof. Since $\omega=0$ if $Q=0$ at a point, we have locally (writing $\omega=p d x$ $+q d y)$ that $p=0$ and $q=0$. On the other hand, writing (1) as a homogeneous uniformly elliptic system for $p$ and $q$, it follows from the BersNirenberg representation theorem that the zeros of such a system are isolated [2].

5. The maximum principle. Our main result is the

THEOREM. The speed $Q$ of a steady, compressible, irrotational subsonic flow $\omega$ on an orientable surface $M$ cannot have a relative maximum at a point of positive Gaussian curvature unless the flow is identically zero on $M$. If a relative maximum is attained at a point $P$ of an open set of zero curvature, then the maximum is attained on the closure of the largest open connected set $\Omega$ of zero curvature which contains $P$ and the flow is parallel in $\Omega$.

Proof. If $Q$ has a relative maximum at a point of some open set $U$ (assumed without loss of generality to lie in a single coordinate patch of $M$ ) and $K \geqslant 0$ on $U$ then $L Q \geqslant 0$ on $U$ by Lemma 1 . By the Hopf maximum principle [4] $Q$ cannot have a maximum in $U$ unless it is constant. If on the one hand, $K>0$ on $U$ then by (8) one sees immediately that $Q \equiv 0$ on $U$ which, by Lemma 2 , is possible only if $Q \equiv 0$ on $M$. On the other hand, if $K=0$ on $\Omega$ then, again from (8), one has $\nabla_{i} \rho \omega_{a}=0$. But $\nabla_{i} \rho \omega_{a}=\rho \nabla_{i} \omega_{a}+$ 
$\omega_{a} \rho^{\prime} \nabla_{i} Q$ and since $\nabla_{i} Q=0$ on $\Omega$ because $Q$ is constant there, we obtain the condition $\nabla_{i} \omega_{a}=0$ that $\omega$ describes a parallel flow in $\Omega$.

As suggested by the theorem, the set on which the speed attains a maximum (unlike the set on which $Q$ vanishes) need not consist of isolated points. In this connection we state the

Corollary 1. Suppose $Q$ attains a nonzero maximum on a subset $\Lambda$ of $M$. Then $\Lambda$ cannot be contained in a neighborhood $N$ of curvature $K \geqslant 0$ unless, in fact, $K=0$ on $N$.

Proof. By Lemma $1, L Q \geqslant 0$ in $N$. Then by the above theorem $Q=$ constant in $N$ which is impossible if $N$ contains a point of curvature $K>0$.

A special case of the theorem is the well-known

COROLlaRY 2. A subsonic plane flow past an obstacle attains its maximum speed on the boundary of the obstacle or at infinity.

In fact, on any surface with boundary whose curvature $K \geqslant 0$, the maximum speed must be attained on the boundary.

6. Examples. (i) The standard torus in $\mathbf{R}^{3}$ is given parametrically by $x=(b+a \cos u) \cos v, y=(b+a \cos u) \sin v, z=a \sin u$ with $0<a<b$ and $0 \leqslant u, v \leqslant 2 \pi$. Its metric tensor is $g_{11}=a^{2}, g_{12}=g_{21}=0, g_{22}=(b+$ $a \cos u)^{2}$ so that $\sqrt{g}=a(b+a \cos u)$. If we prescribe circulation $c$ around the $z$ axis (a representative curve would be $u=$ constant, $0 \leqslant v \leqslant 2 \pi$ ) and zero circulation along a curve $v=$ constant, $0 \leqslant u \leqslant 2 \pi$, then the results of [5] ensure the existence of a unique subsonic $\rho$-harmonic form as long as $0 \leqslant c<c_{\rho}$ for some critical circulation $c_{\rho}$. The speed of the flow tends somewhere to sonic speed as $c \nearrow c_{\rho}$. It is easily checked in this example that the form $\omega=c d v / 2 \pi$ is subsonic $\rho$-harmonic for $c<c_{\rho}$ and has the prescribed circulation. Its speed is given by $Q=c^{2} / 4 \pi^{2}(b+a \cos u)^{2}$ which attains its maximum at $u=\pi$, a circle at every point of which the Gaussian curvature is negative.

Although the results of $\$ 5$ apply only to subsonic flows, in this example for $c=c_{\rho}$ we obtain a flow which is subsonic everywhere except on the "inner" circle $(u=\pi)$ where it is sonic. If $c$ is increased beyond $c_{\rho}$ we first obtain a transonic flow with a region of supersonic flow bounded by sonic lines $\left(u=\pi \pm \theta_{\rho}\right)$ and eventually a flow which is completely supersonic. The maximum is always attained on the circle $u=\pi$.

(ii) Consider now the torus obtained by subjecting the upper half $(z>0)$ of the standard torus to a vertical displacement and then connecting the two halves by right circular cylinders. As above, it is not difficult to write down the $\rho$-harmonic form on the surface thus obtained having the circulations prescribed in example (i). The maximum speed will now be attained on a two dimensional set-the inner cylinder.

ADDED IN PROOF. A more detailed analysis of these examples has been made and will appear in [8]. We consider there any axiallysymmetric torus 
and arbitrary circulations. A complete family of solutions (subsonic, transonic and supersonic) is obtained.

\section{REFERENCES}

1. L. Bers, Mathematical aspects of subsonic and transonic gas dynamics, Wiley, New York, 1958.

2. L. Bers and L. Nirenberg, On a representation theorem for linear elliptic systems with discontinuous coefficients and its applications, Convegno Internaz. Equazioni Lineari alle Derivati Parziali, Edizioni Cremonese, Roma, 1955, pp. 111-140.

3. S. Bochner, Vector fields and Ricci curvature, Bull. Amer. Math. Soc. 52 (1946), 776-797.

4. E. Hopf, Elementare Bemerkungen über die Lösungen partieller Differentialgleichungen zweiter Ordnung vom elliptischen Typus, S.-B. Preuss. Akad. Wiss. Physik-Math. Kl. 19 (1927), 147-152.

5. L. M. Sibner and R. J. Sibner, A non-linear Hodge-de Rham theorem, Acta Math. 125 (1970), 57-73.

6. Non-linear Hodge theory: Compact manifolds and manifolds with boundary, Proc. Summer College on Global Analysis III, Trieste, IAEE Publication, 1974, pp. 121-135.

7. ___ Non-linear Hodge theory: Applications, Advances in Math. (to appear).

8. __ Transonic flow on an axiallysymmetric torus, J. Math. Anal. Appl. (to appear).

Department of Mathematics, Polytechnic Institute of New York, Brooklyn, New YORK 11201

Department of Mathematics, Brookiyn College (CUNY), Brookiyn, New York 11210 